\title{
Including multiple ground motion intensity measures in the derivation of Fragility Functions for Earthquake Loss estimation
}

\author{
Luis Sousa ${ }^{1}$, Vitor Silva ${ }^{2}$, Mário Marques ${ }^{3}$, Helen Crowley ${ }^{4}$ \\ ${ }^{1}$ Department of Civil Engineering, Faculty of Engineering, University of Porto, Porto, Portugal \\ (Imcsousa@fe.up.pt); 2Department of Civil Engineering, University of Aveiro, Aveiro, Portugal \\ (vitor.s@ua.pt); ${ }^{3}$ Department of Civil Engineering, Faculty of Engineering, University of Porto, \\ Porto, Portugal (mariom@fe.up.pt); ${ }^{4}$ European Centre for Training and Research in \\ Earthquake Engineering, Pavia, Italy (helen.crowley@eucentre.it)
}

\begin{abstract}
This paper presents a methodology for the appropriate treatment of variability in the process of building vulnerability assessment. Material, geometric and mechanical properties of the assessed building typologies are simulated through a Monte-Carlo sampling procedure in which the statistical distribution of the latter parameters are taken into account. Record selection is performed in accordance with conditional hazard-consistent distributions of a comprehensive set of intensity measures, and matters of sufficiency, efficiency, predictability and scaling robustness are envisaged in the presented framework. Several intensity measures (IMs) are conjugated in the evaluation of building fragility and vulnerability, whereby fragility functions are established as the multivariate distribution of joint probability of being in a sequential set of damage states. Vulnerability Functions consequently determined provide not only a mean Damage Ratio per level of seismic intensity, but rather probabilistic distributions of Damage Ratio that reflect the ground motion variability expected as the interested site; as determined by the hazard-consistent conditional distribution of a set of sufficient intensity measures.
\end{abstract}

Subject Headings. Civil Engineering, Engineering Structure, Seismic Monitoring Author Keywords. Structural Capacity and Seismic Demand Variability, Ground Motion Selection, Nonlinear Dynamic Analysis, Hazard-Consistent Building Fragility and Vulnerability

\section{Introduction}

The premise that sources of aleatory variability (and its correlation) associated with groundmotion and structural response predictions cannot be neglected in loss assessment procedures has been demonstrated by several authors (e.g. Bazzurro and Luco, 2005). Hence, the purpose of this study is to evaluate and address the appropriate treatment of material, geometric and record-to-record variability in the evaluation of building fragility and vulnerability, whereby several IMs representing measures of spectral intensity, peak ground motion and spectral shape are considered throughout the stages of the proposed framework. Considerations of efficiency (Shome and Cornell, 1999), sufficiency (Luco, 2002), scaling robustness (Kramer and Mitchell, 2006) and predictability (Tothong and Luco, 2007) are thus foreseen in order to ensure the hazard consistent link between structural response and seismic demand.

The General Conditional Intensity Measure (GCIM) approach (Bradley, 2010a) is employed in the selection of natural ground motion records primarily scaled to match different levels of spectral ordinates at the assessed structures' fundamental period of vibration. According to 
the latter, conditional distributions of a relevant set of IMs are determined taking into account all the rupture scenarios influencing seismic hazard at the interested site - Lisbon, Portugal by means of the relative contribution established by disaggregation (Bazzurro and Cornell, 1999) of magnitude, distance and ground motion prediction models (GMPE), as formulated by Lin et al. (2013a).

Thousands of nonlinear dynamic analyses are performed within a probabilistic framework developed by Silva et al. (2014a), where hundreds of reinforced concrete distributed plasticity frame models are simulated in a 2D environment. Through Monte Carlo simulation, variability in geometric and material properties of typical two, five and eight-story pre-code reinforced concrete buildings in mainland Portugal is taken into account. Structural response is assessed in terms of engineering demand parameters (EDP) of peak global drift and inter-story drift, establishing the scope whereby seismic intensity and cumulative percentage of buildings in each damage state are related, by means of a function of multiple IMs.

\section{Numerical Models}

Following the work developed by Silva et al. (2014a), in which material and geometric properties of the most representative Portuguese building typologies were characterized, the numerical models considered herein represent typical buildings constructed before 1958 (pre - seismic code). Dynamic properties are characterized by mean fundamental periods of vibration equal to $0.26,0.45$ and 0.70 seconds, as a result from random generation of assets with respect to geometric and material statistical distributions of two, five and eight story buildings, respectively.

As demonstrated in a study by Silva et al. (2014b), in which a similar sampling framework was implemented, a number of one hundred assets is used to guarantee the statistically significance of the generated distribution of structural capacity. To keep the computational effort in a reasonable level, each structure is modelled as a single infilled frame with three bays, representing the assessed building's lateral load resisting system. Each frame was modelled in 2D environment, with force-based distributed plasticity elements formulation. For the sake of synthesis, considerations adopted with regard to: elements cross section discretization and integration points; material constitutive relationships; p-delta effects; and infill panels modelling approach, are referred to the aforementioned work by Silva et al. (2014a).

\section{Record Selection Methodology}

In the analytical assessment of building fragility and vulnerability, record-to-record variability is of upmost importance, given its influence in the estimated distribution of structural response (Lin et al., 2013b). Amongst the available ground motion selection procedures that incorporate target mean and variance, the Generalized Conditional Intensity Measure (GCIM) approach proposed by Bradley (2010a) is adopted herein, whereby all the intensity measures identified as important to the outcome of the present exercise are foreseen.

It is proposed by the latter that for a given earthquake scenario, or rupture - Rup - any arbitrary vector of ground motion intensity measures - IM - has a multivariate lognormal distribution, to what follows that the distribution of IM given Rup (IM/Rup) conditioned on the occurrence of a particular level of a specific intensity parameter $(I M j)$ $f_{I M i \mid R u p, I M j}\left(\right.$ im $_{i} \mid$ rup $_{k}$, im $\left._{j}\right)$ - presents identical statistical properties. In brief, since additional details shall be referred to the work of Bradley (2010a), upon definition of appropriate Ground Motion Prediction Equations (GMPE) and correlation structure between the different intensity 
measures $(I M i)$ in $I M$, the conditional distribution of each $I M i$ given $I M j=i m j$ is obtained via total probability theorem from:

$$
f_{I M i \mid I M j}\left(i m_{i} \mid i m_{j}\right)=\sum_{k=1}^{N_{R u p}} f_{I M i \mid R u p, I M j}\left(\operatorname{im}_{i} \mid \operatorname{rup}_{k}, i m_{j}\right) P_{R u p \mid I M j}\left(\operatorname{rup}_{k} \mid i m_{j}\right)
$$

Where $f_{I M i \mid I M j}\left(\operatorname{im}_{i} \mid i m_{j}\right)$ is the probability density function - pdf - of IMi given IMj=imj; $f_{I M i \mid R u p, I M j}\left(\right.$ im $\left._{i} \mid \operatorname{rup}_{k}, i m_{j}\right)$ is the pdf of IMi given IMj=imj and Rup=rup $p_{\text {; }}$ and $P_{R u p \mid I M j}\left(\operatorname{rup}_{k} \mid i m_{j}\right)$ is the contribution weight of Rup=rup $p_{k}$, determined through seismic hazard disaggregation. From the assumption that the $I M$ vector is characterized by a multivariate lognormal distribution, it follows that for each $I M i$ in $I M$, $f_{I M i \mid R u p, I M j}\left(\right.$ im $_{i} \mid$ rup $_{k}$, im $\left._{j}\right)$ has a univariate lognormal distribution, which can be defined by its conditional mean and standard deviation parameters (Bradley, 2010a).

\subsection{Probabilistic Seismic Hazard and Disaggregation}

The use of tables within the text should be in a separate line. Each table should be numbered in the caption.

According to the findings of Vilanova et al. (2007), in which the applicability of different GMPEs to the Iberian region has been evaluated, the models developed by Atkinson and Boore (2006) and Akkar and Bommer (2010) are considered herein, with 0.70 and 0.30 logic tree weights, respectively, as defined in the hazard model implemented by Silva et al. (2014c).

Typically, causal earthquake magnitude, source-to-site distance and fault properties are considered in the definition of different scenarios - Rup - contributing to hazard in a given site, as established by disaggregation (Bazzurro and Cornell, 1999). However, following the findings of Lin et al. (2013a), seismic hazard disaggregation is evaluated in terms of Magnitude $(M)$, Distance $(R)$ and $G M P E$, in order to ensure the consistency between target distributions of all considered intensity measures - IMi - in $I M$, and the variability of ground motion properties expected at the interested site - Lisbon, Portugal. Thus, $f_{I M i \mid I M j}\left(\operatorname{im}_{i} \mid \operatorname{im}_{j}\right)$ presented above are estimated for each conditioning intensity level according to the contribution of all $N_{\text {Rup }}$ scenarios and number of GMPES considered:

$$
\begin{gathered}
f_{I M_{i} \mid I M_{j}}\left(\operatorname{im}_{i} \mid \operatorname{im}_{j}\right)= \\
\sum_{m=1}^{N_{G M P E}} \sum_{k=1}^{N_{R u p}} f_{I M_{i} \mid \mathrm{Rup}, I M_{j}}\left(\operatorname{im}_{i} \mid \operatorname{rup}_{k}, \operatorname{im}_{j}, G M P E_{m}\right) P_{R u p, G M P E_{m} \mid I M j}\left(\operatorname{rup}_{k}, G M P E_{m} \mid i m_{j}\right)
\end{gathered}
$$

The Openquake engine (Silva et al., 2014d) which has been used for probabilistic seismic hazard analysis - considering rock site conditions (shear wave velocity in the top $30 \mathrm{~m}$ of the soil of $760 \mathrm{~m} / \mathrm{s}$ ) - does not currently address 3D disaggregation on $M, R$ and GMPE; however, due to its open-source nature, it was possible to produce the necessary intermediate results for the computation of $P_{R u p, G M P E_{m} \mid I M_{j}}\left(r u p_{k}, G M P E_{m} \mid i m_{j}\right)$, as described herein:

$$
P_{R u p, G M P E_{m} \mid I M_{j}}\left(\operatorname{rup}_{k}, G M P E_{m} \mid i m_{j}\right)=\frac{v\left(I M_{j}, R u p \mid G M P E_{m}\right) \cdot P\left(G M P E_{m}\right)}{v(I M j)}
$$

$P\left(G M P E_{m}\right)$ stands for the logic-tree weight assigned to $G M P E_{m} ; v\left(I M j, R u p \mid G M P E_{m}\right)$ is the rate corresponding to the conditional probability of $I M j=i m j$, using $G M P E_{m}$, assuming a Poissonian process; and $v(I M j)$ is the rate of occurrence of $I M j=i m j$, computed from the correspondent rate of exceedance, as proposed by Bradley (2010a).

\subsection{Selected Intensity Measures}

As demonstrated in a study developed by Sousa et al. (2014), in which efficiency of an extensive set of $I M s$ is evaluated in the context of fragility estimation of similar structures, 
intensity measures incorporating velocity and spectral shape characteristics systematically provide higher correlations with damage exceedance probabilities. Any of such indicators can be considered in the GCIM selection; provided that predictability of marginal mean and standard deviation of the logarithm of each IMi, is ensured.

The applicability of the selected GMPEs to the specific case of mainland Portugal, previously addressed in this manuscript, render spectral acceleration at a range of periods as an obvious choice as target IMs. Thus, in order to ensure that target distributions computed for IMs other than spectral ordinates are consistent with the ground motion properties to be expected at the interested site, preference is given to IMs for which marginal median and logarithmic standard deviation can directly be determined or indirectly be inferred from the same GMPEs. Therefore, measures of peak ground acceleration, PGA, peak ground velocity, PGV, acceleration spectrum intensity, ASI, Housner intensity, $\mathrm{HI}$; and spectral ordinates within the range of 0.05 to 3.0 seconds are considered; conditioned on IMj being the spectral acceleration at the assessed structures' fundamental period of vibration $-S a\left(T_{1}\right)$.

For each conditional Magnitude/ Distance / GMPE, median predications of PGA and PGV, along with the associated logarithmic standard deviations, can directly be obtained from the aforementioned GMPEs. As for the case of $A S I$ and $H I$, since both intensity measures result from integrating spectral quantities in the period domain, target distributions can be computed based on the statistical parameters provided for distribution of spectral ordinates, as demonstrated by Bradley (2010b) and Bradley et al. (2009). The correlation models used for all the combinations of IMs considered are summarized in table 1.

\begin{tabular}{c|c|c|c|c|c} 
& $\boldsymbol{S A}\left(\boldsymbol{T}_{\boldsymbol{i}}\right)$ & PGA & PGV & ASI & HI \\
\hline $\boldsymbol{S A}\left(\boldsymbol{T}_{\boldsymbol{i}}\right)$ & $\begin{array}{c}\text { Baker and } \\
\text { Jayaram (2008) }\end{array}$ & $\begin{array}{c}\text { Baker } \\
(2007)\end{array}$ & Bradley (2012) & Bradley (2011) & Bradley (2011) \\
\hline PGA & - & - & Bradley (2012) & Bradley (2012) & Bradley (2011) \\
\hline PGV & - & - & - & Bradley (2011) & Bradley (2012) \\
\hline ASI & - & - & - & - & Bradley (2011) \\
\hline HI & - & - & - & - & - \\
\multicolumn{2}{|c|}{ Table 1: Correlation models considered for application of GCIM methodology }
\end{tabular}

\section{Fragility Assessment Framework}

As discussed by Silva et al. (2014a), the use of local criterion to define limit states when generating fragility curves for population of buildings may not be appropriate. Hence, structural response conditioned on different levels of seismic intensity is evaluated in terms of maximum inter-story drift (ISD) and global drift (GD); defining thresholds of Slight Damage $(S D)$, Moderate Damage $(M D)$, Extensive Damage $(E D)$ and Collapse $(C o l)$ limit states. $G D$ limits are determined according to the evaluation of capacity performed through a displacementbased adaptive pushover (Antoniou and Pinho, 2004). Similarly to what has been considered by other authors, displacement thresholds at each limit state are defined for each sampled frame without masonry infills (bare frame) according to Silva et al. (2014a). The influence of infill panels, which is translated in a significant decrease of displacement capacity, is accounted for by applying the reduction factors proposed by Bal et al. (2010) for each limit state.

To what concerns ISD, a fixed set of values per limit state are defined based on the evaluation of global damage with increasing inter-story drift in 25 dynamic tests performed in real reinforced concrete moment resisting frames by Rossetto and Elnashai (2003). In order to adapt the damage scale proposed by the latter, which includes six levels, to the one presently 
considered, light / slight damage and partial collapse / collapse limit states have been merged, as presented in Silva et al. (2014a).

\subsection{Response Variability and Record selection}

Since records are selected and scaled based on target distributions of a set of IMs that have distinct impacts on the spatial distribution of seismic demand, the number of ground motions required to achieve reasonable confidence in the estimated response's variability is not known a priori. It is recognized in the literature that a large number (greater than thirty) is necessary for the aforementioned purposes (e.g. Lin et al., 2013b); nonetheless, an accurate estimate is highly dependent on the parameters used to characterize response, as well as the structural properties itself.

A number of 150 records, selected according to the GCIM methodology to match target distributions of the $I M$ vector is hereby assumed as a sufficiently large sample to provide an accurate evaluation of inter-story drift (ISD) and global drift (GD) distributions at each level of conditional intensity measure - $S a\left(T_{1}\right)$, in each sampled frame. The minimum number of records necessary to achieve identical distributions within a given statistical significance level can thus be determined by comparing the latter with responses resulting from record sets of increasing size, selected to match the same target $I M$. Accordingly, empirical distributions of $I S D$ and GD obtained from samples of 10 to 140 records are individually compared with the reference sample of 150. This step is performed using the Brown-Forsythe (1974) (BF) test, according to which the hypothesis that two sets of data have equal variance is assessed at the $5 \%$ significance level, as illustrated in Figure 1.

Although only the results pertaining to 5 story frames and $S a\left(T_{1}\right)$ equal to $1.0 \mathrm{~g}$ are presented, the same conclusion is attained for samples of two and eight story frames, at all considered levels of conditioning seismic intensity parameter - $S a\left(T_{1}\right)$. As also illustrated, a value of 60 records is considered to provide an adequate compromise between computational effort and statistical significance of results in terms of variance in distribution of Global Drift and Interstory Drift.
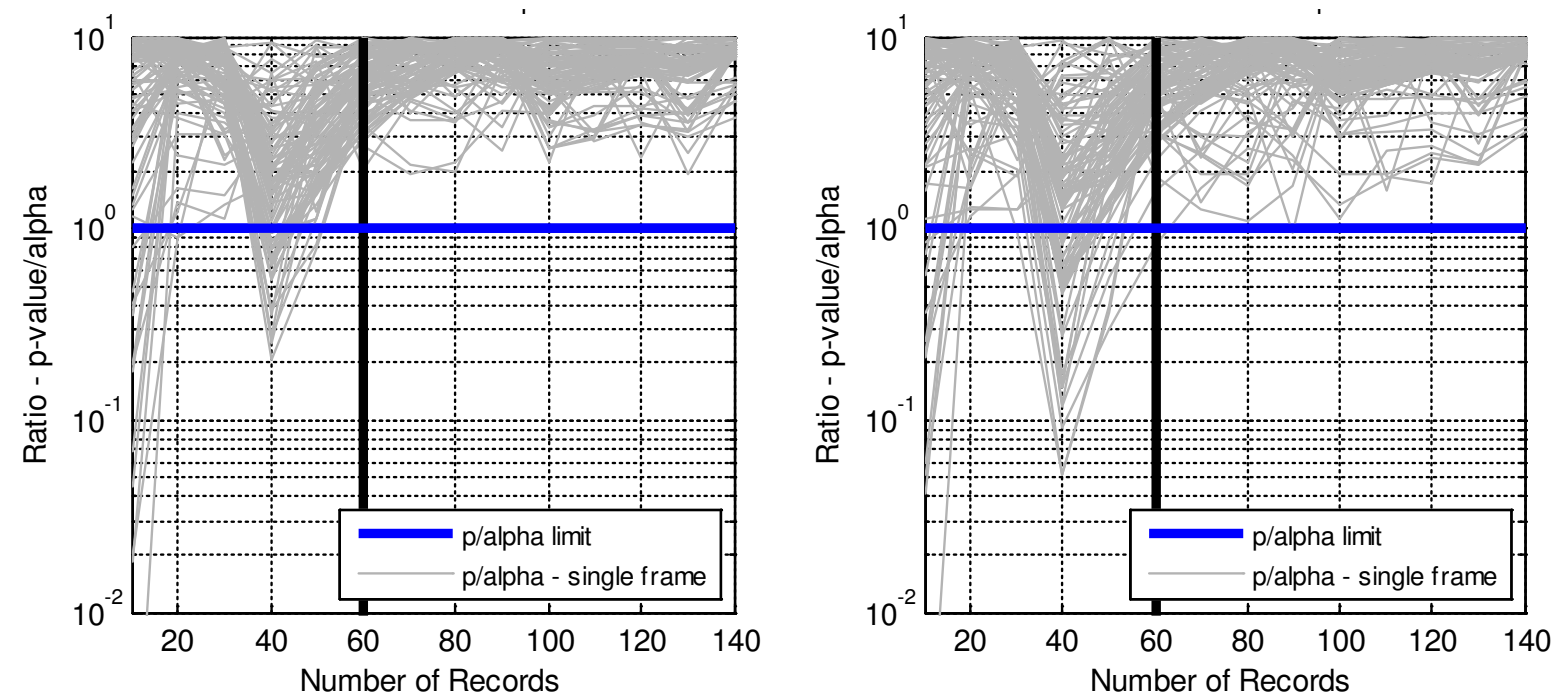

Figure 1: Ratio - $p$-value/alpha - between BF test statistic and corresponding significance level for 100 synthetic 5 story frames, when comparing distributions of response obtained from sets of 10 to 140 records, with the one obtained with the reference set of 150; records selected and scaled to a level of $\mathrm{Sa}(\mathrm{T} 1)=1.0 \mathrm{~g}$. Ratios equal or higher than 1.0 indicate that the null hypothesis of equal variance cannot be rejected at $5 \%$ significance, for GD (left) and ISD (right) 


\subsection{Fragility Functions and Damage state probabilities}

Fragility Functions typically describe the uncertainty in the capacity of a structural system (or a set of synthetically generated buildings, as in the present case), when subjected to sets of ground motion representing increasing values of seismic demand. In this context, it is possible to determine the uncertainty associated with the estimation of probabilities of exceeding specific damage states (for each level of primary intensity parameter - $S a\left(T_{1}\right)=a$ ) through the convolution of the uncertain variables involved: seismic demand and structural capacity, as follows:

$$
f\left[d s_{i \mid S a\left(T_{1}\right)=a}\right]=\int_{I M} F\left[d s_{i_{\mid I M, S a}\left(T_{1}\right)=a}\right] \cdot f\left(I M_{\mid S a\left(T_{1}\right)=a}\right) \cdot d I M
$$

Where $f\left[d s_{i \mid S a\left(T_{1}\right)=a}\right]$ is the probabilistic distribution of probabilities of exceeding limit state $i$ given $S a\left(T_{1}\right)=a ; F\left[d s_{i \mid I M, S a\left(T_{1}\right)=a}\right]$ represents the structural capacity, as the probability of exceeding limit state $i$ given $I M$ and $S a\left(T_{1}\right)=a ; f\left(I M_{\mid S a}\left(T_{1}\right)=a\right)$ is the probability density function of $I M$ given $S a\left(T_{1}\right)=a$; and $I M$ is an intermediate variable that, representing the variability of seismic demand, allows separating the problem of explicitly determining the $f\left[d s_{i_{\mid S a}\left(T_{1}\right)=a}\right]$ distribution, as a conjunction of two uncertain variables: $F\left[d s_{i \mid I M, S a\left(T_{1}\right)=a}\right]$ and $f\left(I M_{\mid S a\left(T_{1}\right)=a}\right)$.

\subsection{Conditional Fragility functions}

As previously presented, $I M$ is the vector of ground motion intensity measures considered in the process of record selection, for which the hazard consistent distribution given $S a\left(T_{1}\right)=a$ $f\left(I M_{\mid S a\left(T_{1}\right)=a}\right)$ - is readily available. However, particular intensity measures are known to perform better in terms of correlation with damage when assessing similar structural typologies (Sousa et al., 2014); leading to the further step of considering only the intensity measure - IMi - for which such correlation is higher (i.e. the most efficient), at each level of $S a\left(T_{1}\right)$. Based on the latter assumption - whose validity is submitted to further investigations - that the most efficient IMi for each level of conditional intensity is sufficient to take into account all the explanatory variables in the $I M$ vector; and given the fact that hazard consistent distributions of $I M i$ given $S a\left(T_{1}\right)=a-f\left(I M i_{\mid S a\left(T_{1}\right)=a}\right)$ - are equally determined in the process of record selection, the formulation presented in Equation 4 is updated, as follows:

$$
f\left[d s_{i_{\mid S a\left(T_{1}\right)=a}}\right]=\int_{I M_{i}} F\left[d s_{i_{\mid I M i, S a\left(T_{1}\right)=a}}\right] \cdot f\left(I M i_{\mid S a\left(T_{1}\right)=a}\right) \cdot d I M_{i}
$$

Where $F\left[d s_{i \mid I M i, S a\left(T_{1}\right)=a}\right]$ translates the probability of exceeding a limit state $i$, as a function of $I M i$, when records are selected and scaled for a particular level of $S a\left(T_{1}\right)$; hereby defined as a conditional fragility function.

As schematically presented in Figure 2, conditional fragility curves are evaluated upon the assumption that "record-specific" distributions of response follow a lognormal probabilistic function. Thus, by computing a distinct exceedance probability for each record, one can additionally assign to it the corresponding record specific value of $I M i$, establishing the conditional relationship between the variables; which enables the use of regression analysis to fit a lognormal cumulative distribution curve, as a function of $I M i$, conditioned on $S a\left(T_{1}\right)=a$ : $F\left[d s_{i_{\mid I M_{i}, S a\left(T_{1}\right)=a}}\right]$. 

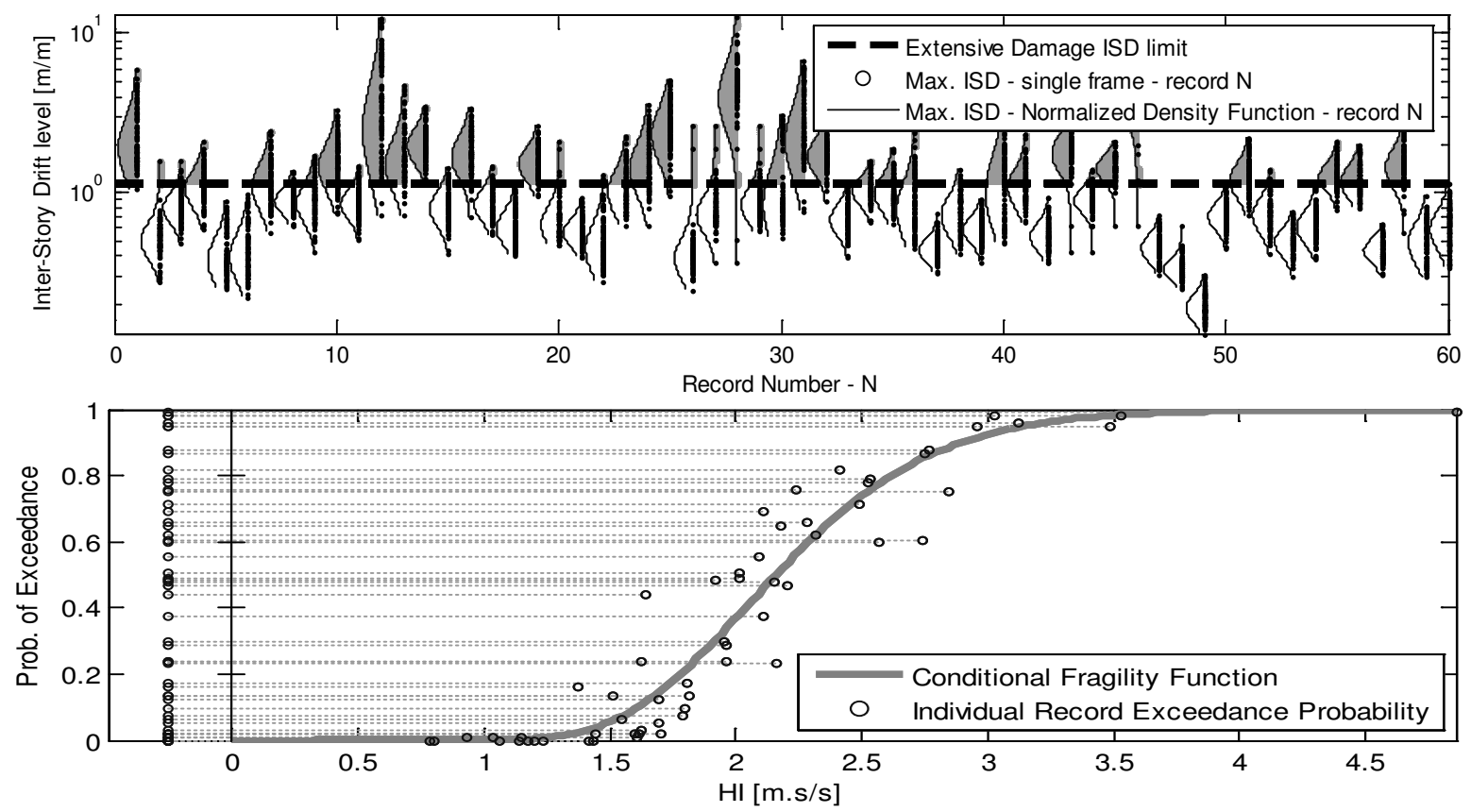

Figure 2: Record-specific distributions of response and corresponding probabilities of exceedance of Extensive Damage, determined according to ISD limit state criteria for 8 story frames (upper). Fitted conditional fragility curve, as a function of $\mathrm{HI}$ (lower). Records selected and scaled for $\mathrm{Sa}(\mathrm{T} 1)=1.0 \mathrm{~g}$

\section{Propagation of uncertainty: from Fragility to Vulnerability}

Given the fact that $f\left[d s_{i \mid S a\left(T_{1}\right)=a}\right]$ (Equation 5$)$ is an uncertain variable, it follows that the distribution of being exactly in a given damage state $i-p\left[d s_{i S a\left(T_{1}\right)=a}\right]$ - is also a random uncertain variant. As outlined in Equations 6 to 10, it can further be demonstrated that its variability is a function of $I M i$; of the uncertainty in the regression of $F\left[d s_{i_{\mid I M_{i}, S a\left(T_{1}\right)=a}}\right]$; and the correlation between uncertainties associated with $F\left[d s_{i \mid I M_{i}, S a\left(T_{1}\right)=a}\right]$ at different limit states:

$$
p\left[d s_{i_{\mid S a\left(T_{1}\right)=a}}\right]=\int_{I M_{i}=0}^{+\infty} P\left[d s_{i_{\mid I M_{i}, S a\left(T_{1}\right)=a}}\right] \cdot f\left(I M_{\left.\right|_{\mid S a}\left(T_{1}\right)=a}\right) \cdot d I M_{i}
$$

Where $P\left[d s_{i \mid I M_{i}, S a\left(T_{1}\right)=a}\right]$ is the probability of being exactly in damage state $i$, conditioned on $I M i$ and $S a\left(T_{1}\right)=a$; as determined by following Equations 7 and 8:

$$
\begin{aligned}
& P\left[d s_{i_{\mid I M_{i}, S a\left(T_{1}\right)=a}}\right]=F\left[d s_{i_{\mid I M_{i}, S a\left(T_{1}\right)=a}}\right]-F\left[d s_{i+1 \mid I M_{i}, S a\left(T_{1}\right)=a}\right] ; 1 \leq i \leq N \\
& P\left[d s_{i_{\mid I M_{i}, S a\left(T_{1}\right)=a}}\right]=F\left[d s_{i_{\mid I M_{i}, S a\left(T_{1}\right)=a}}\right] ; i=N
\end{aligned}
$$

In which $N$ is the number of defined limit states and $F\left[d s_{i \mid I M_{i}, S a\left(T_{1}\right)=a}\right]$ is the conditional probability of exceedance of limit state $i$, defined by the corresponding conditional fragility function.

According to the formulation above, it follows that the mean and variance of $p\left[d s_{i_{\mid S a}\left(T_{1}\right)=a}\right]$ depends on the mean and standard deviation of $F\left[d s_{i \mid I M_{i}, S a\left(T_{1}\right)=a}\right]$, which, as subsequently 
presented (and aforementioned), depends on the correlation between regression uncertainties associated with $F\left[d s_{i \mid I M_{i}, S a\left(T_{1}\right)=a}\right]$ at different limit states:

$$
\begin{aligned}
& \mu_{P\left[d s_{i \mid I M i, S a\left(T_{1}\right)=a}\right]}=\mu_{F\left[d s_{\left.i_{\mid I M i, S a\left(T_{1}\right)=a}\right]}\right]}-\mu_{F\left[d s_{i+1 \mid I M i, S a\left(T_{1}\right)=a}\right]} \\
& \sigma_{P\left[d s_{i \mid I M i, S a\left(T_{1}\right)=a}\right]}^{2}=\sigma_{F\left[d s_{i \mid I M i, S a\left(T_{1}\right)=a}\right]}^{2}+\sigma_{F\left[d s_{i+1 \mid I M i, S a\left(T_{1}\right)=a}\right]}^{2}-2 \times \\
& \operatorname{COV}\left\{F\left[d s_{i_{\mid I M i, S a}\left(T_{1}\right)=a}\right], F\left[d s_{i+1_{\mid I M i, S a}\left(T_{1}\right)=a}\right]\right\}
\end{aligned}
$$

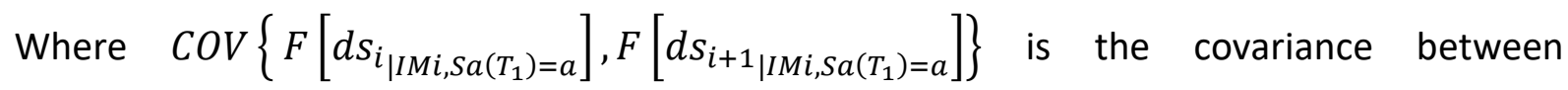
distributions of damage exceedance probability for damage states $i$ and $i+1$, conditioned on a particular level of $I M i$ and $S a(T 1)=a$.

\subsection{Combining sources of uncertainty}

In order to completely define: a) the probabilistic distribution of $p\left[d s_{i \mid S a\left(T_{1}\right)=a}\right]$; and b) $P\left[d s_{0_{\mid S a\left(T_{1}\right)=a}}, d s_{\left.\right|_{\mid S a\left(T_{1}\right)=a}}, d s_{2_{\mid S a}\left(T_{1}\right)=a}, d s_{3_{\mid S a}\left(T_{1}\right)=a}, d s_{4_{\mid S a}\left(T_{1}\right)=a}\right]$, the joint distribution of damage probabilities; in which $d s_{0}$ to $d s_{4}$ refer to damage states of No Damage (ND), SD, MD, $E D$ and $\mathrm{Col}$, by the same order; Equations 6 to 10 are numerically solved through a MonteCarlo simulation procedure, whereby a set of probabilities of being exactly in damage states of $N D, S D, M D, E D$ and $C o l$ are computed for each simulation of IMi on the dIMi domain.

As illustrated in Figure 3, a bootstrap method has been used for computing a set of 500 conditional fragility curves, for each limit state. More importantly, each synthetic dataset has been primarily generated for the first limit state and the associated data points for the remaining limit states were selected in accordance with the latter. In these circumstances, it is possible to identify which conditional fragility curves - for limit states of $S D, M D, E D$ and $C o l$ - correspond to which bootstrap simulation. Thus, for each Monte-Carlo simulation of IMi on the dIMi domain, 500 damage probabilities of $S D, M D, E D$ and $C o l$ are jointly determined, according to Equations 7 and 8 . The final result of the simulation procedure schematically presented in Figure 3 is thus a matrix of $M \times N$ dimension, in which $M$ corresponds to the total number of simulations ( $1000 M C$ simulations times 500 bootstrap samples) and $N$ refers to the number of damage state probabilities evaluated (ND, SD, MD, ED and Col). Therefore, the latter matrix constitutes a sampled approximation to the joint distribution of $P\left[d s_{0 \mid S a\left(T_{1}\right)=a}, d s_{1_{\mid S a\left(T_{1}\right)=a}}, d s_{2 \mid S a\left(T_{1}\right)=a}, d s_{3 \mid S a\left(T_{1}\right)=a}, d s_{4 \mid S a\left(T_{1}\right)=a}\right] ;$ hereby referred as "empirical simulated" joint distribution. 

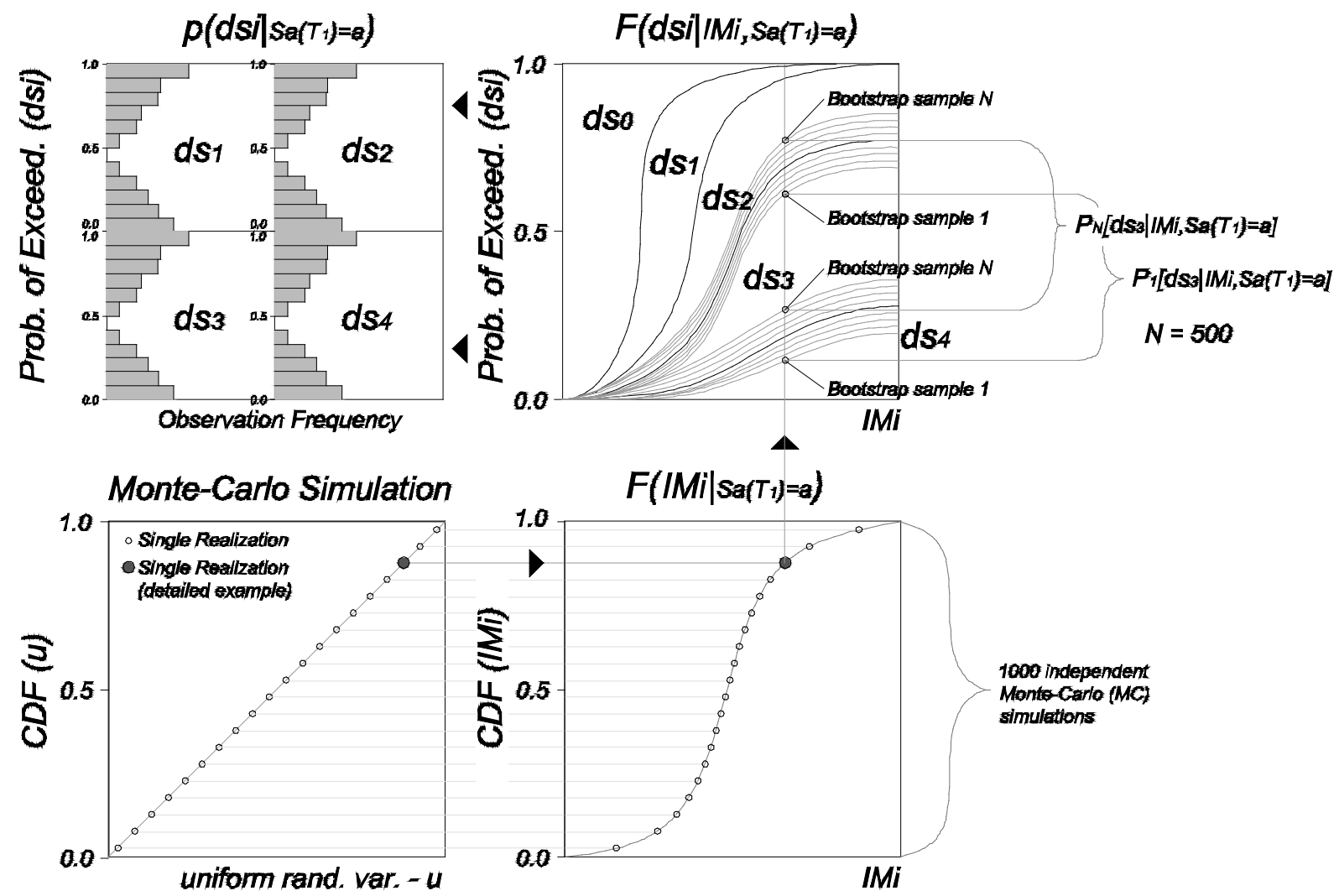

1000 independent

Monte-Cario (MC)

simulations

Figure 3: Schematic representation of numerical solution of Equation 6,

through Monte-Carlo sampling on the distribution of IMi given Sa(T1)=a

Fragility functions are thus hereby established as the multivariate model of joint probability of being in damage states of Slight Damage, Moderate Damage, Extensive Damage and Collapse. Therefore, one can make use of the "empirical simulated" joint distribution; resorting to any appropriate Consequence Function to associate a Damage Ratio to each of the jointly simulated damage state probabilities. For illustration purposes, the Consequence Models proposed by Silva et al. (2014a), Bal et al. (2008) and FEMA-443 have been used. Damage ratio distributions are determined, according to the aforementioned methodology, for levels of $S a\left(T_{1}\right)$ ranging from $0.1 \mathrm{~g}$ to $1.0 \mathrm{~g}$, for 2,5 and 8 story buildings; considering InterStory and Global Drift damage criteria; as depicted in Figure 4, for the case of ISD. 

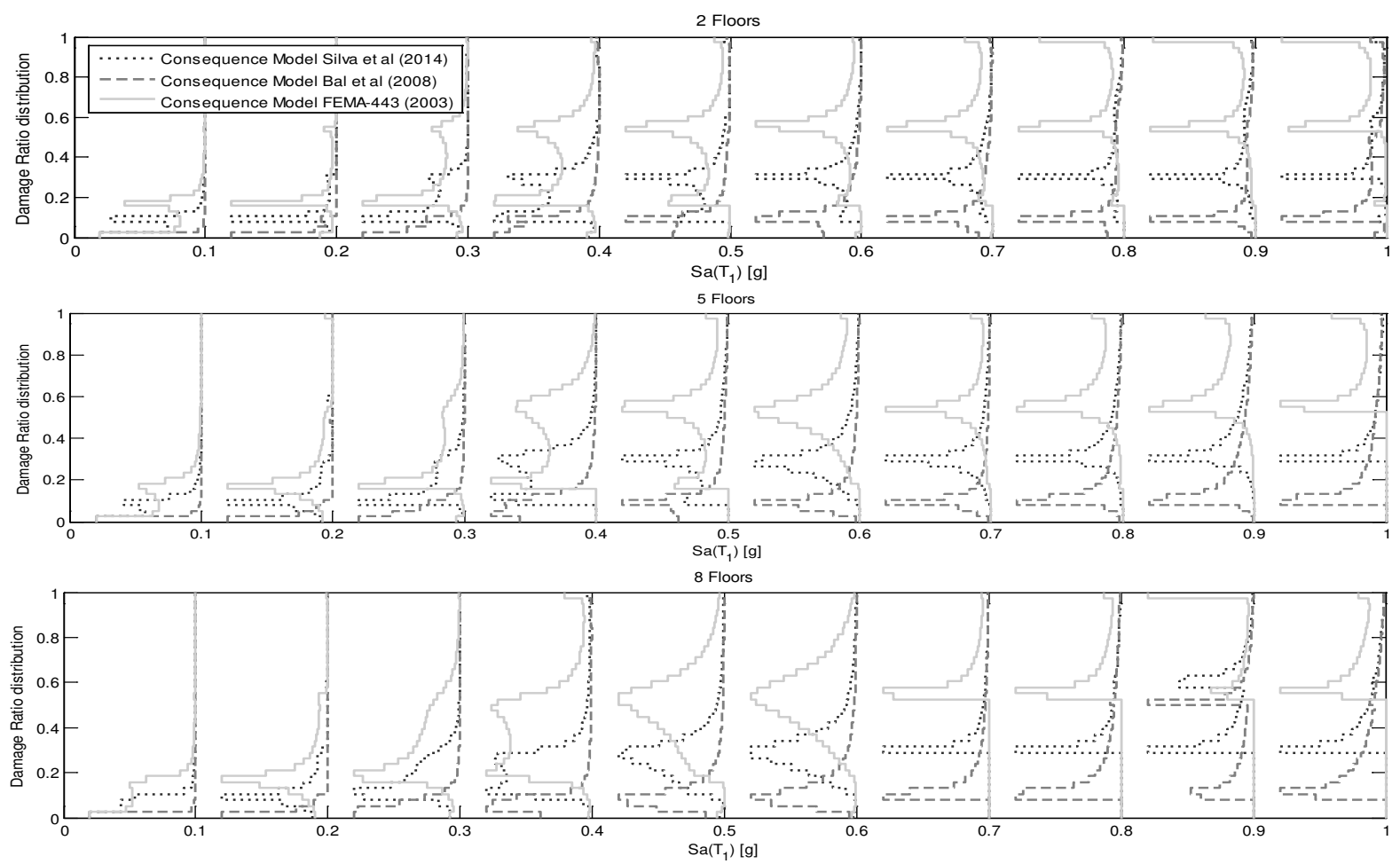

Figure 4: Empirical distribution of Damage Ratio for levels of Sa(T1) ranging from $0.1 \mathrm{~g}$ to $1.0 \mathrm{~g}$. Inter-Strory drift Criteria. 2, 5 and 8 Story frames

The Vulnerability Functions consequently determined provide not only a mean damage ratio, but rather distributions of damage ratio per level of $S a\left(T_{1}\right)$ that reflect the ground motion variability expected at the interested site for such level of intensity; as determined by the hazard-consistent conditional distribution of a sufficient IMi.

\section{Conclusions}

Variability in structural capacity has been properly addressed through a probabilistic approach towards the modelling of material, geometric and mechanical properties of two, five and eight story buildings constructed in Portugal before 1958 (pre-code). The Generalized Conditional Intensity Measure approach was adopted for the purpose of record selection, reflecting the variability of peak ground acceleration, PGA, peak ground velocity, PGV, acceleration spectrum intensity, ASI, Housner intensity, $\mathrm{HI}$; and spectral ordinates within the range of 0.05 to 3.0 seconds, conditioned on increasing levels of spectral acceleration at the assessed structure's fundamental period of vibration.

Based on the appraised minimum number of ground motion records required to achieve reasonable confidence in the estimated responses' variability, the concept of conditional fragility functions has been introduced, translating the probability of exceeding a set of damage states as a function of a sufficient IMi, when records are selected and scaled for a particular level of the primary intensity measure: $S a\left(T_{1}\right)$. In this context, IMi is an intermediate variable that, representing the variability of seismic demand, allows separating the problem of determining the distribution of damage exceedance probabilities, as a conjunction of two uncertain variables: structural capacity represented by the conditional fragility function and seismic action characterised by the hazard consistent distribution of $I M i$, conditioned on the interested level of $S a\left(T_{1}\right)$.

Similarly, it has been demonstrated that the use of IMi as an intermediate variable allows the simulation of joint probabilities of being in a sequential set of damage states. Thus, Fragility 
functions are hereby established as the multivariate model of joint probability of being in damage states of Slight Damage, Moderate Damage, Extensive Damage and Collapse. The latter allows the use of any Consequence Function to obtain Vulnerability functions characterized by distributions of damage ratio that reflect the uncertainty due to the ground motion variability expected at the interested site, for each level of primary intensity parameter - $S a\left(T_{1}\right)$.

\section{References}

Akkar, S., Bommer. J. 2010. "Empirical equations for the prediction of PGA, PGV and spectral accelerations in Europe, the Mediterranean region and the Middle East". Seismological Research Letters; 81(2):195-206.

Atkinson, G., Boore, D. 2006. "Earthquake ground-motion prediction equations for eastern North America". Bulletin of the Seismological Society of America; 96(6):2181-2205.

Baker J.W. 2007. "Correlation of ground motion intensity parameters used for predicting structural and geotechnical response". Application of Statistics and probability in Civil Engineering; ISBN 9780415451345.

Baker J.W. and Jayaram, N 2008. "Correlation of Spectral Acceleration Values from NGA Ground Motion Models2. Earthquake Spectra; Vol. 24, No. 1, pp. 299-317.

Bal, I.E., Crowley, H., Pinho, R., Gulay, F. 2008. "Detailed assessment of structural characteristics of Turkish RC building stock for loss assessment models". Soil Dynamics and Earthquake Engineering, Vol. 28, pp. 914-932.

Bal, I. E., Crowley, H., Pinho, R. 2010. “Displacement-based earthquake loss assessment: Method development and application to Turkish building stock". ROSE Research Report 2010/02; IUSS Press, Pavia, Italy.

Bazzurro, P. and C. A. Cornell. 1999. "Disaggregation of seismic hazard". Bulletin of the Seismological Society of America; 89(2), 501-520.

Bazzurro, P. and Luco, N. 2005. "Accounting for uncertainty and correlation in earthquake loss estimation". 9th International Conference on Structural Safety and Reliability (ICOSSAR'05); Rome, Italy.

Bradley BA, Dhakal RP, Cubrinovski M, MacRae GA. 2009. "Ground-Motion Prediction Equation for SI based on Spectral Acceleration Equations". Bulletin of the Seismological Society of America; 99:277-285.

Bradley BA. 2010a. "A generalized conditional intensity measure approach and holistic ground-motion selection". Earthquake Engineering \& Structural Dynamics; 39(12):13211342. DOI: 10.1002/eqe.995.

Bradley BA 2010b. "Site-specific and spatially distributed Ground-Motion prediction of Acceleration Spectrum Intensity". Bulletin of the Seismological Society of America; Vol. 100, No. 2, pp. 792-801. DOI: 10.1785/0120090157.

Bradley BA. 2011. "Empirical Correlation of PGA, spectral accelerations and spectrum intensities from active shallow crustal regions". Earthquake Engng Struct. Dyn; 40:17071721

Bradley BA. 2012. "Empirical Correlations between Peak Ground Velocity and Spectrum-Based Intensity Measures". Earthquake Spectra: Vol. 28, No. 1, pp. 17-35

Brown, Morton B. Forsythe, Alan B. 1974. "Robust tests for equality of variances". Journal of the American Statistical Association; 69: 364-367. DOI: 
Kramer SL, Mitchell RA. 2006. "Ground motion intensity measures for liquefaction hazard evaluation". Earthquake Spectra; 22(2):413-438.

Lin, T., Harmsen S., Baker, J., Luco, N. 2013a. "Conditional Spectrum Computation Incorporating Multiple Causal Earthquakes and Ground-Motion Prediction Models". Bulletin of the Seismological Society of America. April; 103:1103-1116.

Lin, T., Haselton, C. B., Baker, J. 2013b. "Conditional - Spectrum - based ground motion selection. Part II: Intensity-based assessments and evaluation of alternative target spectra". Earthquake Engineering \& Structural Dynamics. DOI: 10.1002/eqe.2337.

Luco N. 2002 "Probabilistic seismic demand analysis, connection fractures, and near-source effects". Ph.D. Thesis; Department of Civil and Environmental Engineering, Stanford University, 2002; 285.

Rossetto, T., Elnashai, A. 2003. "Derivation of vulnerability functions for European-type RC structures based on observational data". Engineering Structures; 25(10), 1241-1263.

Shome N, Cornell CA. 1999. "Probabilistic seismic demand analysis of nonlinear structures". Technical Report RMS-35; RMS Program: Stanford CA.

Silva, V., Crowley, H., Pinho, R., Varum, H., Sousa, L. 2014a "Investigation of the characteristics of Portuguese regular moment resisting frame RC buildings and development of a vulnerability model". Bulletin of Earthquake Engineering.

Silva, V., Crowley, H., Pinho, R., Varum, H., Sousa, R. 2014b. "Evaluation of analytical methodologies used to derive vulnerability functions". Earthquake Engineering \& Structural Dynamics, 2014; 43:181-204. DOI: 10.1002/eqe.2337.

Silva, V., Crowley, H., Pinho, R., Varum, H. 2014c. "Seismic Risk Assessment for mainland Portugal". Bulletin of Earthquake Engineering.

Silva, V., Crowley, H., Pagani,M., Monelli, D., Pinho, R. 2014d. "Development of the OpenQuake engine, the Global Earthquake Model's open-source software for seismic risk assessment". Natural Hazards; DOI: 10.1007/s11069-013-0618-x.

Sousa, L., Silva, V., Marques, M., Crowley, H., Pinho, R. 2014. "Including multiple IMTs in the development of fragility functions for earthquake loss estimation". Proceedings of the 2nd International Conference on Vulnerability and Risk Analysis and Management; Liverpool, United Kingdom.

Tothong P, Luco N 2007. "Probabilistic seismic demand analysis using advanced ground motion intensity measures". Earthquake Engineering and Structural Dynamics; 36(13):1837-1860.

Vilanova, S., Fonseca, J. F. B. D., Oliveira, C. S. 2007. “Ground-Motion Models for Seismic Hazard Assessment in Western Iberia: Constraints from Instrumental Data and Intensity Observations". Bulletin of the Seismological Society of America; vol. 97 no. 5 1702-1717. DOI: $10.1785 / 012005019$.

\section{Acknowledgements}

This work has been performed within the framework of the research project PTDC/ECMEST/3062/2012 'Earthquake loss assessment of the Portuguese building stock' funded by the Foundation of Science and Technology (FCT) of Portugal. 